ORCID: $\underline{0000-0002-0529-6010}$

\title{
Similarities Between the AdS/CFT Correspondence and the Binding Problem
}

\begin{abstract}
Michael T. Elliott
Abstract - The binding problem refers to the puzzle of how the brain combines objects' properties such as motion, color, shape, location, sound, etc., from diverse regions of the brain and forms a unified subjective experience. Holographic physical systems, recently discovered darlings of theoretical physics, began with research into black holes but have since evolved into the study of condensed matter systems in the laboratory like superfluids and superconductors. A primary example is the AdS/CFT correspondence. A recent conjecture of this correspondence suggests that holographic systems combine information from across a boundary surface, sort out the simplest description of said information, and, in turn, use it to determine the geometry of spacetime itself in the interior - a kind of geometric hologram. Although we would never tend to think of these two processes as related, in this paper we point out ten similarities between the two and show that holographic systems are the only physical systems that match the subjective and computational characteristics of the binding problem.
\end{abstract}

\section{Introduction}

As long ago as the fourth century B.C., the ancient Greek scholar Hippocrates suggested the seat of conscious perception was located in the brain (Bear, 2015). Today, it is still a mystery what gives rise to the phenomenon of perception. The mammalian visual cortex has now been completely mapped and we know that no full representation of the external world exists at the neuronal level (Feldman, 2013). Still, much has been learnt. We know "color and sounds, for instance, are processed separately from the moment they hit our sensory receptors, while others, like color and shape, are initially encoded together but subsequently processed in different regions of the brain" (Goldstein, 2010; Holcomb, 2009). Despite the separation of processing, and the lack of a full representation, the brain must figure out which features correspond to which objects (visual feature binding) and construct a unified perception of the external world (known as the subjective unity of experience). This is the binding problem.

Various approaches, such as Feature Integration Theory (FIT), have attempted to describe a plausible way in which the brain might computationally associate various features from distant regions, like color, shape, and sound with objects (see Feldman, 2013; Robertson, 2005). In such an approach, simulated neurons combine higher-and-higher level features in progressively deeper layers leading to neurons that detect specific objects. This approach is consistent with neurological observations, too, where certain neurons are known to fire exclusively in response to the detection of specific individuals - so-called "grandmother" neurons (Martindale, 2005). However, if we follow this approach to try to explain the unity of experience, 
then we must ultimately integrate all the features of all relevant objects perceived at a given moment into one neuron, and then must locate the seat of conscious perception in these neurons. In this scheme, all relevant perceptual information is being brought to that neuron and being perceived there. However, this seems implausible both on the basis of the combinatorial explosion that results from all possible situations (Stiefel, 2014) and the awkwardness of locating the seat of perception in individual, constantly changing neurons.

Alternatively, some have suggested temporal synchrony causes binding (von der Malsburg, 1981). It is known that neuron firings capturing information associated with a common object are synchronized temporally (see Holcomb, 2009). However, there are two problems: synchronization occurs much too slowly to explain the results of binding experiments (Feldman, 2013), and, two, there seemingly must be at least some role for spatial proximity and other factors because it is clearly implausible to believe that everything that occurs with approximate simultaneity has a shared subjective experience. So, it is not generally viewed that temporal synchrony alone can solve the binding problem (Feldman, 2013; Dong, 2008).

Recently, Hierarchical Predictive Coding (HPC) models, from the same family of models as deep learning neural networks (DLNNs), have received great interest (Spratling, 2016). These models are motivated by the observation that the brain is not passively interpreting information, but actively constructing an image of the external world. This internal image encodes prior experience, and allows predictions to be made, which generate prediction errors which, in turn, are used to improve the model's ability to identify objects and their properties under adverse conditions (Spratling, 2016).

Still, while the tenets of HPC's seem reasonable enough, and the approach has demonstrated its merits in computer object recognition models (Spratling, 2016), the problems these models are trying to solve have been shown to have exponential complexity (Kwisthout, 2014), which means the computational burden of solving them remains intractable by any classical computational process (including any neuron level computation based on classical physics) (see Aaronson, 2005, pg. 3). Furthermore, these models only address the computational aspects of binding, they don't suggest anything to address the origin of subjective experience (Feldman, 2013).

Meanwhile, on the other side of science, in the seemingly unrelated field of theoretical physics, it has been an exciting couple of decades for physicists. The first glimpse of how quantum field theory may be unified with gravity, a so-called "theory of everything", has been discovered under the heading of the holographic principle (Musser, 2018; Maldacena, 1997). The most prominent example of the holographic principle is known as the AdS/CFT correspondence (Anti-de Sitter space/Conformal Field Theory) and came from imagining a hypothetical black hole embedded in a toy model of the Universe. While the AdS/CFT model does not describe the large-scale geometry of this Universe, it has successfully provided an understanding of phenomena that would otherwise defy explanation (e.g. high temperature superconductivity).

Generally, the idea of holography is that there is a correspondence between quantum field theory on the "boundary" surface enclosing a region of spacetime, and spacetime geometry in the interior "bulk". The boundary can be thought of as creating a holographic gravitational image in the bulk in one higher dimension, hence the name. So, a 2-D surface would create a 3-D image in the bulk. Further, every single quantity on the boundary corresponds to a dual quantity in the bulk (Czech, 2017). We can do a calculation using quantum field theory on the boundary without gravity, or we can do a gravity calculation in the bulk without quantum field theory and always get the same answer (Susskind, 2014). Sometimes problems that are very difficult to solve in one form can be translated into a simple, easy-to-solve form in the other (for an overview, see Cowen, 2015; Van Raamsdonk, 2010; Moskowitz, 2016; more technical: Harlow, 2018). Interestingly, however, AdS/CFT not only has implications for hypothetical black holes, but has profound implications for almost everyday systems here on earth in the areas of condensed matter and nuclear physics (Hubeny, 2015).

Physicists are developing a dictionary of these dual quantities, mapping the boundary quantum physics to the bulk gravity physics (see Zaanen, $2015 \mathrm{pg}$. 175 for a list of dictionary entries). While much remains to 
be learnt about holography, a real game-changer occurred in 2014 when physicist Leonard Susskind conjectured a new relationship for this dictionary. The idea was that the geometry of spacetime in the bulk was shaped by the complexity of the quantum information on the boundary (Susskind, $2014 \mathrm{~B}$; for an overview see Cole, 2015).

Quantum information is encoded in qubits which are the quantum analogue of bits in a classical computer (Li, 2001). Just as any arbitrary information, like an image or a sentence, can be encoded in the bits of a computer, so, too, can it be encoded in qubits. Qubits can store much more information than bits, however, because they can take advantage of the quantum principles of superposition and entanglement. A computer circuit is a sequence of logical gates (like AND, OR, NOT, etc.) that can be used to construct any arbitrary string of bits, and, likewise, a quantum circuit can compose any arbitrary quantum state of qubits. Quantum circuits can replicate any computation that can be done in a classical circuit and hence they are universal computing processes (Renka, 2018).

What makes Susskind's conjecture a real game-changer is that complexity refers to the simplest possible quantum circuit that could produce the quantum information. Specifically, 'simplest possible' means the smallest number of quantum gates. In general, there will be a huge number of possible quantum circuits that can produce the information but only the simplest is relevant for determining spacetime's geometry in the bulk.

We can illustrate the profundity of this proposal with a simple toy problem - a Rubik's cube. A Rubik's cube can be in some 43 quintillion different configurations (Rokicki, 2014), but this is utterly trivial compared to the number of configurations of the qubits on the boundary of a holographic system like a black hole. Even a simple system like the cube has too many possible combinations to try them all, even on a supercomputer, and so it took about 30 years after the Rubik's cube emerged as a popular toy in 1980's for its complexity to finally be proved. It turns out to have a maximum of 20 (Rokicki, 2014). That is, any configuration of the cube can be constructed in 20 turns of the cube or less. Stated in reverse, we can solve any configuration of the cube in 20 turns or less. By analogy, in a purely hypothetical scenario, if a qubit model of the cube was represented on the boundary of a blackhole, the geometry inside would keep changing as the cube became more and more mixed (assuming starting from a solved state). Specifically, the volume of spacetime behind the horizon would keep growing in proportion to the complexity, but then would stop growing as the number of turns reached 20 . The blackhole would figure out the simplest way to describe the cube and that would cap its growth. It is stunning that such a basic physical system is solving this sort of problem, but that is the claim of this conjecture. Scott Aaronson, computer scientist, put it this way: "...surely we can't expect the laws of physics to know about nontrivial efficient algorithms like (say) Edmonds' algorithm for maximum matching, or the ellipsoid algorithm for convex programming. Yet those algorithms could in principle be the reason why a state had polynomial rather than exponential quantum circuit complexity" (Aaronson, 2016 in reference to the growth rate, polynomial or exponential with time, of volume inside a black hole).

Since its proposal, Susskind's conjecture has withstood some non-trivial tests (Aaronson, 2016). The most relevant of which, for our purposes, apply to systems that occur here on Earth in the laboratory: superfluids, and superconductors. These systems are characterized by extensive quantum entanglement which means that they behave totally unlike a system of individual particles and like a single giant particle (Minkel, 2009). The mathematics to describe these quantum systems is so complicated it defies solution. However, it turns out that they follow something called an 'entanglement entropy area law' which means the same holographic physics are in play that describe a black hole (Herdman, 2016). Moreover, it means the Ads/CFT dictionary is applicable and that includes Susskind's conjecture. Modeling the superfluid as a sort of black hole in a hypothetical Universe, allows physicists to transform the difficult mathematics of the quantum state on the boundary into a simple, solvable gravity problem in the bulk. For instance, when a superconductor goes through a transition from insulator to superconducting phase, the complexity of quantum entanglement in the system spikes (Momeni, 2016; Ghodrati, 2018). The equations are intractable from the perspective of the quantum field theory but solvable within the framework of general relativity. This 
allows these systems to be modeled in a very elegant and tractable way, and, importantly, produces results consistent with known experiments in the lab (Ball, 2017; Asano, 2016; Gooth, 2017; Zhang, 2018; Wenger, 2012).

While the mathematics is solvable and giving the right answers, the challenge with holography is figuring out how to make sense of what the physics is saying. For example, in (Adams, 2012) the authors locate the superfluid on the boundary of an AdS spacetime. Sure enough, the gravitational dual allows for vortices in the superfluid to be understood and modeled by the physics in the bulk. Physicist Hong Liu put it this way: "these very complicated quantum mechanical collective effects are beautifully captured by black hole physics. For strongly correlated systems [like superfluids], if you put an electron into the system, it will immediately 'disappear' - you can no longer track it. It's like an object falling into a black hole." Wolchover, 2013. But the whole setup is a bit troubling because 1,) this Universe does not have AdS geometry (it appears to be flat!) (NASA, 2014), and 2.) the superfluid is existing right here in the lab, not on the faraway edge of a spacetime manifold. So, holography needs some generalization beyond AdS/CFT to have a full theory consistent with the real world. The belief among practitioners is that the principles of holography apply to all gravitational spacetimes, but that we only know how to apply it presently to AdS ones. (Zaanen, 2015, pg. 11). In other words, quantum entanglement on any boundary surface drives the interior bulk spacetime geometry regardless of its location and irrespective of the large-scale geometry of the Universe (see Susskind, 2017, Miyaji, 2015, Dong, 2018 for research generalizing the ideas of holography beyond AdS/CFT; also, see [Figure 1.b] as an illustration of how entanglement and spacetime imply one another).

\section{Parallels Between Subjective Perception and Holographic Systems}

What gives rise to the unity in the subjective unity of experience? What combines information from throughout the brain? And, what binds this information together to synthesize objects and their properties in a single continuous stream of perception? These are the sorts of questions we will address in this section. We will argue that for each property of subjective perception there is a dual physical property of holographic systems. For example, we will discuss a holographic property whose physical description is quite similar to the subjective characteristic of unity, another holographic property that combines information from distant regions, and another that finds simplifying structure in the information, etc. All told we will discuss 10 characteristic qualities of perception that are remarkably similar to properties of holographic systems; a list that is by no means exhaustive. We will thus make a 10 -fold circumstantial argument (i.e. if it walks like a duck, talks like a duck, etc.) that holography, as in holographic superfluidity, gives rise to subjective perception and, thereby, binding. Further, we will show that holographic systems are the only known physical systems that mirror these subjective qualities.

In brief, we will suppose superfluidity occurs in the brain; we will locate the seat of our conscious perception in the shoes of spacetime itself in the bulk of this superfluid. Perceptual data will play the role of boundary information. Then, we will argue, the physical process of spacetime sorting out the complexity of this information to determine its own geometry is the same process as subjectively perceiving it - a subjective/physical duality. We will follow this up with a plausibility argument demonstrating how superfluidity could be achieved in the brain, and, last, will discuss implications for the behavior of superfluids in the laboratory.

\section{What property of holographic systems corresponds to unity?}

Quantum entanglement is a physical process that takes two or more qubits (whether the qubits be photons, electrons, or much larger systems) and makes them into one quantum system. Paraphrasing Einstein, it is 
possible to have all possible knowledge about a system, and yet, this does not include any information about its individual parts. It is literally as though individual things have given up their individual identity, if you will, and become one thing. In a mathematical sense, the quantum state cannot be written as a product of the individual quantum states of the constituents; it cannot be factorized into parts. The mathematical wave function that describes the system has become inseparable. Clearly this is a process whose physical description offers some interesting parallels to subjective unity. The idea of the importance of quantum entanglement in binding is not a new one; see, for instance, (Marshall, 1989). In a holographic physical system, all the qubits on the boundary are entangled together and form one quantum system.

Subjective perception is experienced as a unified reality; spacetime geometry in the bulk is determined by the unified quantum information on its boundary.

\section{What holographic process can combine information from distant regions?}

Information is combined from all over the brain, including color, shapes, sounds, smells and integrated into one whole subjective experience. In a holographic system, all the information on the boundary collectively determines the geometry of spacetime in the bulk ( $\underline{\mathrm{Fu}, 2018})$.

Information stored throughout the brain is combined to form subjective perception; spacetime is combining information all over its boundary to shape itself.

\section{What holographic process takes all this unified, non-local information and comes up with a simple description of it?}

The idea that the brain is integrating prior knowledge with sensory information to find the most probable explanation in subjective perception is wide spread in neuroscience (Kwisthout, 2014). To infer the most probable explanation, however, research in algorithmic information theory suggests the brain must find the simplest internal model of the perceptual information; simplicity is equivalent to reality (Ruffini, 2017). Spacetime is doing the same sort of thing: it must find the simplest description of the information on its boundary to determine its quantum circuit complexity. This complexity will, in turn, determine the geometry of spacetime (Susskind, 2014).

Subjective perception sorts out the simplest (most likely) presentation of all relevant perceptual information, while spacetime must find the simplest description of quantum information.

4. Spacetime must find the simplest description alright, but how do we know that this description has an object structure like subjective perception? For instance, how do we know that the simplest quantum circuit contains something like a grandmother?

In order to compute the complexity of the quantum information on the boundary, spacetime shapes itself into a geometry that decodes this information. DLNN's, too, are models designed to identify the patterns in information. When a DLNN is trained to learn the features of the boundary, remarkably, the network geometry that results (network structure, weights) mirrors the geometric structure of spacetime (You, 2017). The depth of the neural network corresponds to the distance into the bulk from the boundary, and the connection weights correspond to the metric of spacetime (a specific component of its geometry) (Hashimoto, 2018). Both processes begin by detecting simple local features of the information. This occurs in the lower layers of the neural net and in spacetime near the boundary containing the quantum information. As we proceed to deeper layers of the neural network, and to the geometry deeper inside the bulk, both reflect detection of more complex, non-localized features (Bear, 2015, pg. 363; You, 2017). This suggests that spacetime is sorting out the patterns in the same way that a DLNN would (for an overview see 
Wolchover, 2014) and, so, if a grandmother feature was hypothetically encoded in the weights of a DLNN it would be similarly encoded into the metric of spacetime.

Perhaps another useful way to think of this process is to imagine decomposing a bitmap image (large file size) into the vector graphics objects that comprise it (small file size). A DLNN's lower layers detect edges in the image, higher layers detect the objects present; the vector graphics representation is much lower complexity than the bitmap (smaller file size). Spacetime does the same sort of thing.

Thus far, this research has concentrated on a variety of basic physical systems ranging from blackholes to molecules of Sm0.6Sr0.4MnO3 to nuclear physics (Hashimoto, 2018B) with tremendous success. And, while none of these systems are as complicated as the information in the brain, both DLNN's and spacetime geometry appear to be general models of learning.

Spacetime produces the same sort of geometry as a neural network in sorting out the complexity of the boundary information. Neurons in deeper layers of DLNN's respond to specific objects.
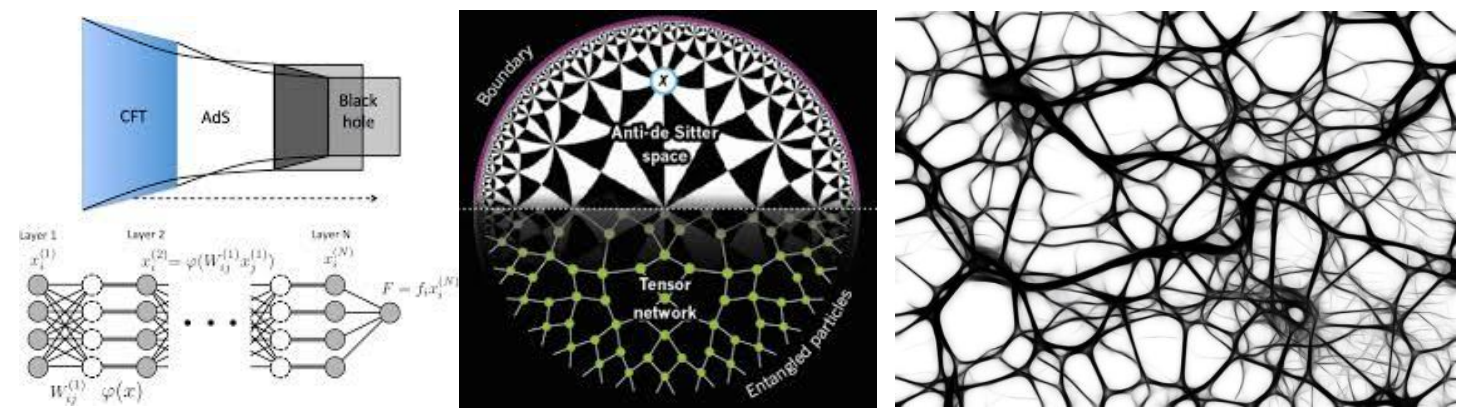

Figure 1. Three things that appear far more inter-related than previously thought: spacetime geometry, quantum information, and subjective perception. (Left) a,Deep Learning Neural Network geometry (network structure, weights) and the geometry of spacetime in AdS/CFT are related. Both are learning the patterns and structure of information. (from "Deep learning and the AdS=CFT correspondence"). (Middle) b.the duality between spacetime geometry and quantum information, (from R.Cowen, "The quantum source of space-time", Nature 527 (2015) 290-293). (Right) c.Neurons in the brain (from "The Sounds Inside Your Brain Right Before Death").

5. Perception is based not only on sensory information but prior experience. It is widely believed prior experience is stored in the neural network architecture of the brain. Is it possible to translate the information contained in neural networks into a quantum circuit?

Prior experience is thought to be encoded in the firing patterns of neurons (Rosenblatt, 1958). If prior experience influences perception, as it is thought to do, and perception is to be based on quantum information, then there must be a means by which neural information is translated into the quantum kind.

Recent research has shown how the sort of neural information and functionality, as modeled in a DLNN, can be embedded in a quantum circuit (Schuld, 2014). All the features of the DLNN, like the layers of neurons, the connection weights between them, and their activation functions are replicated in the quantum circuit. Moreover, and unique to the quantum version, the entire population of input data (i.e. training data) can be loaded simultaneously so that the quantum version of a neural network exists in a superposition of evaluating multiple input data at once. As will be seen shortly, this is an important feature because it opens the door to the possibility of finding a globally optimal set of weights that best fits the input data. It represents an advantage over classical approaches to training DLNN's that follow gradient descent procedures based on one input example at a time. To conceptualize this, we may imagine the input data to be a sequence of still frames capturing perceptual information, then a superposition of such frames could be characterized as a perceptual stream like a movie. The whole perceptual stream is available for learning simultaneously, 
rather than learning from one frame at a time. Interestingly, the subjective experience of perception has been compared to an internal movie before in (Chalmers, 2014).

It is possible to capture the information and structure of a classical neural network in a quantum circuit.

6. The task of associating properties with objects in the most likely way is exponentially hard (lots of local optima), but subjective perception occurs seamlessly. What physical process can handle this computational burden?

Is it just window dressing that holography brings to the table? Adding a fancy subjective movie-like experience to matter but not adding anything computationally? That is, is there anything going on in binding that cannot be computationally simulated on a conventional computer? Research suggests that the task of prediction error minimization in HPC is NP-hard (Kwisthout, 2014) as is the task of finding an optimal configuration of a DLNN (Blum, 1992). The NP-hard complexity class means that the length of time it takes to find the global solution grows exponentially with the number of inputs. So, finding the optimal configuration of a DLNN with $n$ inputs grows as $C^{\wedge} n$ where $C$ is a constant. For large $n$, as are probably involved in perception, this renders the problem completely intractable.

This is in stark contrast to subjective experience where it certainly feels like we are successfully finding a global minimum (the simplest explanation). That is, we all successfully perceive the same reality in front of us most of the time. This is true even in challenging landscapes; for example, consider people's ability to consistently recognize CAPTCHA images, while computer algorithms cannot (von Ahn, 2000). In special cases, when there is not a distinct global minimum, like when the simplest explanation is ambiguous, a remarkable situation results known as multistability, e.g. the Necker cube or the Rubin vase (Baars, 2010, chapter 5.1, or the now famous 'dress' illusion on social media (

Because the computational tasks of HPC and deep learning are NP-hard, because the subjective evidence suggests individuals are perceiving a common global solution, and, given the size of the problem - the visual cortex alone has $n>1$ billion neurons (Azevedo, 2009) - it suggests binding is solving a problem that no classical computational process can solve efficiently. That goes for any process constructed based on the classical laws of physics, be it silicon circuits, electro-chemical interactions in neurons or otherwise (Aaronson, 2005). Spacetime on the other hand, in determining the quantum circuit complexity of the information on the boundary, is efficiently solving a known NP-hard problem ( $\underline{\mathrm{Nam}, 2018})$.

In the previous section we discussed how a stream of perceptual information from a neural network could plausibly be encoded in a quantum circuit. Holography gives us a means to efficiently find the simplest description of that perceptual stream, like a holographic movie encoded in the geometry of spacetime.

Perception finds the simplest description of the external world (reality) amidst a non-linear landscape of many local optima (i.e. the computational problem is NP-hard); spacetime solves the simplest quantum circuit problem which is also known to be NP-hard.

\section{Perception occurs in real-time. Even though quantum circuit complexity can be efficiently calculated, can it be solved in real-time?}

Black hole dynamics are extremely fast; a solar-mass black hole thermalizes (reaches thermal equilibrium) in about a millisecond (Susskind, 2018). While this is happening spacetime must solve the complexity of the boundary to determine the volume (geometry) behind the black hole's horizon. That means that a black hole can solve the complexity of the boundary quantum state in less than a millisecond, and probably far 
less. The point here is that the spacetime dynamics of holographic systems are fast enough to enable the perception of reality in real-time.

Subjective perception orchestrates binding in real-time; spacetime computes complexity in real-time too.

8. Perception is stable, but quantum information is notoriously susceptible to decoherence. How can these two things then be related?

Perception is remarkably stable from one moment to the next. Quantum systems are notoriously fragile, so any discussion linking perception to quantum information must include a substantial provision for quantum error correction. Turns out this is the case in holographic physical systems. Since the state of the qubits distributed across the entire boundary collectively imply the geometric structure of subregions of spacetime in the bulk, holographic systems are naturally error correcting (Almheiri, 2014; for an intro see Wolchover, 2019). This works intuitively in the same sense as a classical holographic image. There, because the holographic film encodes the image in a manner that distributes the information across the entire film, if a portion of the film is destroyed, the holographic image is still preserved.

Perception is stable. Holographic systems are naturally error-correcting. Even when boundary information is destroyed, redundancy stabilizes the holographic geometry in the bulk.

9. Perception is relatively simple despite perceptual information often being complex. Does holography facilitate this?

In the perception of color, for example, we know that the physical wavelength of light alone is insufficient to account for perceived color (see Stone, 2012, Chapter 1 for some illustrative examples) and depends in a complicated way on the particular scene that is perceived. The brain must integrate complex raw perceptual information with prior experience. In holographic systems, complex information on the boundary can translate into a simple geometric description in the bulk (Aaronson, 2016). In other words, spacetime is already putatively doing the heavy lifting of transforming the complex boundary information into a simple hologram in the bulk; all that remains is to apply a relatively simple mapping of the hologram onto the subjective experience of perceiving it.

The translation from boundary information to bulk geometry is not complexity preserving. Complex problems on the boundary can translate to simple problems in the bulk.
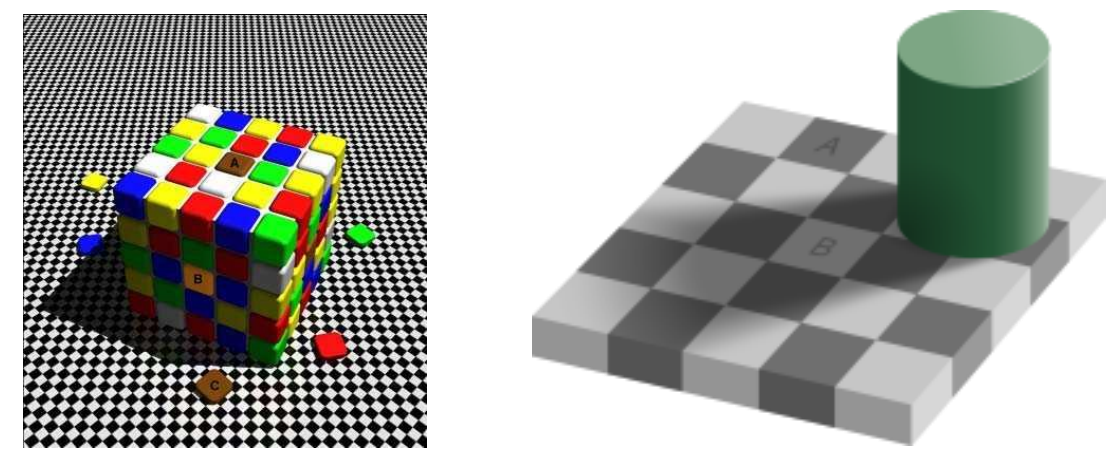

Figure 2. Perception depends in a complicated way on visual information. Wavelength of light alone is not sufficient to explain the perception of color. (Left)Tiles A, B, \& C are all the same color (from "Color Cube"). (Right) Squares $A$ \& $B$ are the same shade (from "Checker Shadow Illusion"). 
10. Perception is translationally, rotationally and scale invariant. It is not known how the visual cortex does this, however. Where does this capability come from?

Neurons exist in the visual cortex that fire in response to objects regardless of their location in the visual field, their rotation (viewing angle), and their scale (large or small) (Sountsov, 2011; Ito, 1995). Moreover, humans consistently demonstrate this ability despite not being trained to recognize objects from different angles and at different scales (Sountsov, 2011). How did the brain develop these capabilities? The neural structure that gives rise to them is not known (Sountsov, 2011).

DLNN's, by contrast, do not learn symmetries like these on their own as a result of training; they must be either (a.) encoded in the model prior to training by including, say, special convolutional layers (translational invariance), or (b.) must be guided by a pre-processing step that replicates training images to be presented at many different angles of rotation and scales ( $\underline{\mathrm{Xu}, 2014} ; \underline{\mathrm{Li}, 2017})$.

Holographic systems, on the other hand, are finding a global solution when they solve the circuit complexity problem. And, since there are known quantum circuits that can handle translation, rotation, and scale invariance (Grant, 2018), if such circuits participate in the simplest description of boundary information, spacetime would be expected to discover them. This would likely be the case in a Universe such as ours where these symmetries are commonplace.

There are known quantum circuits that are invariant under translation, rotation, and scale transformations and spacetime in holographic systems can be expected to discover them in the solving the circuit complexity problem.

\section{Table 1. Ten Parallels Between Subjective Perception and Holographic Physical Systems (AdS/CFT)}

\begin{tabular}{|c|c|c|}
\hline Label & $\begin{array}{c}\text { Subjective Perception } \\
\text { 1.Unity }\end{array}$ & $\begin{array}{c}\text { Holographic Physical System } \\
\text { (AdS/CFT) }\end{array}$ \\
\hline 2.Non-locality & $\begin{array}{c}\text { Object's properties processed in diverse } \\
\text { regions of the brain }\end{array}$ & $\begin{array}{c}\text { boundary information is unified by } \\
\text { quantum entanglement }\end{array}$ \\
\hline 3.Complexity & $\begin{array}{c}\text { perceived reality is the simplest } \\
\text { description of perceptual information } \\
\text { the whole boundary }\end{array}$ & $\begin{array}{c}\text { the simplest description determines } \\
\text { spacetime geometry }\end{array}$ \\
\hline $\begin{array}{c}\text { 4.DLNN Geometry } \\
\text { = Spacetime } \\
\text { Geometry } \\
\text { information that become increasingly } \\
\text { complex and non-local in deeper layers }\end{array}$ & $\begin{array}{c}\text { spacetime geometry does the same } \\
\text { thing; network weights equate to the } \\
\text { spacetime metric, deeper layers } \\
\text { equate to depth in the bulk }\end{array}$ \\
\hline $\begin{array}{c}\text { 5.Prior Experience } \\
\text { prior experience stored in neurons }\end{array}$ & $\begin{array}{c}\text { neural network information can be } \\
\text { converted into quantum circuits }\end{array}$ \\
\hline 6.Computability & perception is probably an exponential \\
complexity problem (NP-Hard) & $\begin{array}{c}\text { spacetime solves an NP-Hard problem } \\
\text { efficiently }\end{array}$ \\
\hline 7.Dynamics & perception/binding occurs real-time & $\begin{array}{c}\text { spacetime solves complexity in real- } \\
\text { time }\end{array}$ \\
\hline 8.Stability & perception is stable & $\begin{array}{c}\text { holographic systems implement error- } \\
\text { correcting codes to stabilize } \\
\text { information }\end{array}$ \\
\hline $\begin{array}{c}\text { 9. Complexity } \\
\text { preserving }\end{array}$ & $\begin{array}{c}\text { perception is simple while perceptual } \\
\text { information is complex, e.g. wavelength }\end{array}$ & $\begin{array}{c}\text { holographic systems are not } \\
\text { complexity preserving; complex }\end{array}$ \\
\hline
\end{tabular}




\begin{tabular}{|c|c|c|}
\hline & $\begin{array}{c}\text { alone cannot explain the perception of } \\
\text { color }\end{array}$ & $\begin{array}{c}\text { information on the boundary can } \\
\text { translate into a simple description in } \\
\text { the bulk }\end{array}$ \\
\hline $\begin{array}{c}\text { 10. Translation, } \\
\text { rotation, and scale } \\
\text { invariance }\end{array}$ & $\begin{array}{c}\text { perception of objects is reliable } \\
\text { regardless of position, rotation, or scale }\end{array}$ & $\begin{array}{c}\text { quantum circuits exist, and would be } \\
\text { discovered, that are translation, } \\
\text { rotation, and scale invariant and would } \\
\text { be discovered in holographic systems }\end{array}$ \\
\hline
\end{tabular}

\section{Holography is the *Only* Way}

In this section we argue that holographic systems are the only known physical systems that mirror the subjective and computational characteristics of perception. For starters, quantum entanglement is the only process known in physics by which multiple independent qubits (of any kind) can be unified in a physical way. The mathematical description of quantum entanglement results in an inseparable quantum state description. There is nothing in the classical (non-quantum) description of physics that groups a collection of objects, be it particles, atoms, molecules, etc., and physically identifies them as one thing. So, without quantum entanglement we would need to draw an arbitrary line in the sand somewhere (e.g. that all molecules vibrating at the same frequency have unified perceptions, or all neurons within a few centimeters of some central location, etc.). Quantum entanglement can be used to explain non-locality too, as it occurs across the entire boundary of a holographic system. Quantum entanglement alone is not enough, however, as it does not capture the complexity aspect of subjective experience. Subjective perception is about finding the simplest description of perceptual information and therefore we require a physical process that does this and does it efficiently and in real-time.

We've already discussed why such a problem could never be efficiently simulated by any classical process, however, could such a problem be solved by a quantum computer? Quantum computers can solve some problems in the category of NP-hard, but only those problems that have special structure where we can use interference effects to manipulate the qubits into producing a solution - like in Shor's algorithm for factoring integers (Shor, 1995). Unfortunately (or fortunately), the quantum circuit complexity problem is not thought to be efficiently solvable by a quantum computer ( $\underline{\mathrm{Nam}, 2018)}$ which suggests a quantum computer could not solve the binding problem. And, since quantum computers have the proven ability to simulate all pure quantum systems (Lloyd, 1996), this means no purely quantum process can solve the binding problem, either. Holographic systems are the only known physical systems with this capability and do so because they exist outside the purely quantum world where spacetime couples to quantum information in profound ways (like Susskind's conjecture).

Is it possible that perception could be linked to another complexity problem, like the Kolmogorov complexity? It is certainly possible, but this complexity problem is even more difficult, falling in the category of the uncomputable (Chaitin, 1995). Other complexity problems, like classical circuit, communication, decision tree, and quantum communication complexity are all thought to not be efficiently solvable by a quantum computer (O'Donnell, 2015). Therefore, not only are holographic systems the only ones that mirror the subjective characteristics of perception, they are the only known physical systems that are capable of solving the binding problem.

\section{Discussion}

\section{Biological Plausibility}

The above arguments suggest some sort of holographic physical system in the brain, like a superfluid, but how could such a state of matter occur there? There have been a number of recent proposals that argue 
for quantum effects in the brain - for a review see (Jedlicka, 2017). However, calculations have demonstrated that fast decoherence rates should be expected in such an environment (Tegmark, 1999) and that flies in the face of highly entangled systems like superfluids. How is it possible to overcome such objections? While the details of a theory of holographic superfluidity in the brain are beyond the scope of this paper, we will explore some particularly relevant possibilities for the purpose of demonstrating plausibility, and for suggesting future research.

Interestingly, recent experiments have demonstrated that a kind of long-range entangled quantum state known as a Fröhlich condensate does indeed form in a biological protein crystal under exposure to a $\mathrm{THz}$ laser (Lundholm, 2015). A Fröhlich condensate is like a Bose-Einstein Condensate (BEC), which has been identified with superfluidity (Leighton, 1959). While such a state was proposed as a source of biological quantum coherence more than 50 years ago by Herbert Fröhlich, their detection has remained elusive until recently. Their discovery was made possible only because of recent advancements in $\mathrm{THz}$ laser technology (Lundholm, 2015). THz is a region of the electromagnetic spectrum particularly relevant to biological systems because this frequency range interacts strongly with the low frequency vibrations of common biomolecules like DNA and proteins. In order for a Fröhlich condensate to explain superfluidity in the brain, evidence of a metabolic source that could provide this pumping energy to sustain the condensate in vivo and in lieu of the THz laser would need to be found.

Beyond metabolic considerations, because the holographic boundary must include diverse regions of the brain associated with color, smell, shapes, etc., it would be necessary for the superfluid state to exist in widespread regions of the brain simultaneously. Interestingly, Fröhlich showed how a condensate could form throughout an entire biological organ in his work (Fröhlich, 1977); see (Hyland, 2015, pg. 196-198) for an overview of this work). Also, conspicuously, research continues to discover a great number of effects of the THz spectrum on biological function such as DNA transcription (González-Jiménez, 2016), regulating stem cell growth (Bock, 2010), effects on cell membrane permeability (Perera, 2019), and more (see Zhao, $\underline{2014}$ for an overview).

Of course, a means of interaction between the superfluid and a macroscopic system of neurons would be required. The amplification of quantum level effects to the macro scale has been proposed before via the neurological mechanism of cellular ion channels (Vaziri, 2010; Jedlicka, 2017). These are individual proteins that only allow a single ion to pass into or out of neurons at a time and are small enough to be influenced by quantum scale effects, yet able to affect neuron firing rates at macro scales and vice-versa.

\section{Superfluid Helium}

In this paper we have described a number of parallels between the subjective experience of binding and the objective characteristics of holographic systems like superfluids. But, if this connection is useful in understanding the phenomena of binding, shouldn't it be useful in the other direction as well? In other words, if perception is linked to superfluidity, then shouldn't we expect superfluid helium, for instance, to exhibit behavior that would demonstrate at least some primitive form of perception? Indeed, studies of superfluid helium reveal very deep, complex, remarkable behavior that could be characterized as life-like.

Liquid helium undergoes a transition to a superfluid state when it is cooled down to near absolute zero, 2.17K (Schmitt, 2014). In this state it exhibits several remarkable behaviors. First, when contained in a beaker, it will spread a microscopically thin film across all accessible surfaces. It will then flow up, over and down the walls of the beaker if and only if it can reach a lower energy gravitational state outside (Golovko, 2011). There are other fluids that will climb walls, but these occur due only to local osmotic pressure; there are no global considerations driving the fluid's actions. The action of a superfluid, on the other hand, could be characterized as deliberate, and purposeful. It could even be considered to perceive its surroundings and take actions based on a kind of global awareness. 
When droplets of a normal fluid are spun in a centrifuge they will eventually fly apart as the speed of rotation is increased. This action has the capability to destroy a superfluid too. However, a superfluid exhibits the remarkable ability to withstand a much higher rate of rotation then a normal fluid (Gomez, 2014; see Diep, 2014 for an introduction). It does this by forming vortices that counter-balance the centrifugal stress and allow it to remain fluid until much higher rotational velocities. Heat, also, will destroy a superfluid if its temperature is raised enough to cause it to undergo a phase transition back to the state of an ordinary fluid. Remarkably, when exposed to a heat source, a superfluid will form a dynamic flowing fountain (Allen, 1938). It does this to recirculate and dissipate the heat flowing into it from the source. This action allows it to cool itself and remain in a state of superfluidity. Both actions can, in some sense, be characterized as clever, but, perhaps more fittingly, they can be characterized as a primitive sort of survival instinct. Without these innovative solutions, the entanglement within the superfluid would be broken and the superfluid would be destroyed.

\section{Conclusion}

In this paper we have proposed a novel solution to the binding problem that unites two fields at opposite ends of scientific research. We show the subjective and computational characteristics of binding are remarkably similar to the properties of holographic physical systems like superfluids where the principles of AdS/CFT are applicable. Furthermore, holographic systems are the only known physical systems that have such properties. In order to connect holography with biology, we have suggested a Fröhlich condensate as a source of superfluidity in the brain and cited the recent experimental discovery of such a condensate in biological proteins.

In a sense holographic physics is a natural place to suppose the existence of a dictionary mapping physical properties to subjective ones; we have discussed how spacetime is already putatively doing the heavy lifting of translating complex boundary information into a simple geometric holographic description in the bulk (as in the CFT->AdS dictionary), so it is a natural extension to suppose a secondary, relatively simple translation of the holographic image to subjective perception (like AdS->subjective perception).

Still, many open questions remain, and a detailed theory connecting holography and subjective perception is needed. For instance, what exactly is the superfluid in the brain and what comprises its boundary? Do neurons play a role in encoding quantum information? If so, how does that process work? Is neural geometry replicating holographic spacetime geometry? If so, how does spacetime geometry physically influence neural geometry? Is the holographic bulk geometry related to the internal model of HPC? Is temporal synchrony a way of directing the loading of quantum information onto the holographic boundary and thereby focusing attention? Ultimately, what are the detailed entries in the holographic geometry>subjective perception dictionary? Whatever the answers to these questions may be, we hope to have presented a convincing high-level argument that superfluidity and holographic physics are needed to understand perception and the binding problem and that we have framed some very compelling and interesting possibilities for future research. 


\section{$\underline{\text { References }}$}

Aaronson, S. (2005) NP-complete Problems and Physical Reality. arXiv:quant-ph/0502072

Aaronson, S. (2016) The Complexity of Quantum States and Transformations: From Quantum Money to Black Holes. arXiv:1607.05256v1 [quant-ph]

Adams, A., Chesler, P., Liu, H. (2012) Holographic Vortex Liquids and Superfluid Turbulence.

arXiv:1212.0281v2 [hep-th]

Allen, J. (1938) Superfluidity II — the fountain effect. Nature 141, 243-244 (1938)

Almheiri, A., Dong, X., Harlow, D. (2014) Bulk Locality and Quantum Error Correction in AdS/CFT. JHEP 1504:163,2015

Asano, Y., Filev, V., Kováčik, S., O'Connor, D. (2016) A Computer Test of Holographic Flavour Dynamics II. JHEP03(2018)055

Azevedo, F., Carvalho, L., Grinberg, L., Farfel, J., Leite, R., Jacob Filho, W., Lent, R., Herculano-Houzel, S. (2009) Equal numbers of neuronal and nonneuronal cells make the human brain an isometrically scaled-up primate brain. J Comp Neurol. 2009 Apr 10;513(5):532-41. doi: 10.1002/cne.21974

Baars,B., Gage, N. (2010) Cognition, Brain, and Consciousness. https://doi.org/10.1016/B978-0-12375070-9.00017-6

Ball, P. (2017) Big Bang gravitational effect observed in lab crystal. Nature News

Bear, M. F., Connors, B. W., \& Paradiso, M. A. (2015). Neuroscience: Exploring the brain. Baltimore, Md: Lippincott Williams \& Wilkins.

Blum, A., Rivest, R. (1992) Training a 3-Node Neural Network is NP-complete. Neural Networks

Volume 5, Issue 1, 1992, Pages 117-127. https://doi.org/10.1016/S0893-6080(05)80010-3

Bock, J., et.al. (2010) Mammalian Stem Cells Reprogramming in Response to Terahertz Radiation. PLoS ONE 5(12): e15806. https://doi.org/10.1371/journal.pone.0015806

Chaitin, G., Arslanov, A., Calude, C. (1995) Program-Size Complexity Computes the Halting Problem. CDMTCS Research Report Series

Chalmers, D. (2014) How do you explain consciousness? .TED Talk Online

Cole, K. (2015). Wormholes Untangle a Black Hole Paradox. Quanta Magazine

Cowen, R. (2015). The quantum source of space-time . Nature News

Czech, B. (2017). Einstein Equations from varying Complexity. Phys. Rev. Lett. 120, 031601 (2018)

Diep, F. (2014) The Weird Ways Of Superfluid Helium. Popular Science

Dong, Y., Mihalas, S., Quu, F., von der Heydt, R., \& Niebur, E. (2008). Synchrony and the binding problem in macaque visual cortex. Journal of vision, 8(7), 30.1-16. doi:10.1167/8.7.30 
Dong, X., Zhou, L. (2018) Spacetime as the optimal generative network of quantum states: a roadmap to

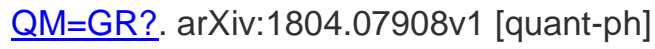

Feldman J. (2012). The neural binding problem(s). Cognitive neurodynamics, 7(1), 1-11.

Fröhlich, H. (1977) Long-Range Coherence in Biological Systems. Riv. Nuovo Cim. 7: 399.

https://doi.org/10.1007/BF02747279

Fu, Z., Maloney, A., Marolf, D., Maxfield, H., Wang, Z. (2018) Holographic Complexity is Non-Local.

10.1007/JHEP02(2018)072

Ghodrati, M. (2018) Complexity growth rate during phase transitions

Goldstein, E. B., (2010). Encyclopedia of Perception. SAGE Publications, Inc

Golovko, V. (2011) Why does superfluid helium leak out of an open container?. arXiv:1103.0517

[physics.flu-dyn]

Gomez, L. et. al. (2014) Shapes and vorticities of superfluid helium nanodroplets. Science 22 Aug 2014:Vol. 345, Issue 6199, pp. 906-909 DOI: 10.1126/science.1252395

Gooth, J., et. al. (2017) Experimental signatures of the mixed axial-gravitational anomaly in the Weyl semimetal NbP. Nature 547, 324 10.1038/nature23005

González-Jiménez, M., Ramakrishnan, G., Harwood, T., Lapthorn, A., Kelly, S., Ellis, E., Wynne,K. (2016) Observation of coherent delocalized phonon-like modes in DNA under physiological conditions. Nature Communications volume 7, Article number: 11799

Grant, E., Benedetti, M., Cao, S., Hallam, A., Lockhart, J., Stojevic, V., Green, A., Severini, S. (2018) Hierarchical quantum classifiers. npj Quantum Information 4, 65 10.1038/s41534-018-0116-9

Harlow, D. (2018). TASI Lectures on the Emergence of the Bulk in AdS/CFT. arXiv:1802.01040 [hep-th

Hashimoto, K., Sugishita, S., Tanaka, A., Tomiya, A. (2018) Deep Learning and AdS/CFT.

Phys. Rev. D 98, 046019 (2018) 10.1103/PhysRevD.98.046019

Hashimoto, K., Sugishita, S., Tanaka, A., Tomiya, A. (2018B) Deep learning and holographic QCD. Phys. Rev. D 98, 046019 (2018) https://doi.org/10.1103/PhysRevD.98.106014

Herdman, C., Roy, P., Melko, R., Maestro, A. (2016) Entanglement Area Law for Superfluid 4 He. Nature Phys. 13, 556 (2017) 10.1038/nphys4075

Holcomb, A.O. (2009). The Binding Problem. Online

Hubeny, V. (2014). The AdS/CFT Correspondence. arXiv:1501.00007v2 [gr-qc]

Hyland, G. (2015) Herbert Fröhlich - A Physicist Ahead of His Time`.https://doi.org/10.1557/mrs.2016.19

Ito, M., Tamura, H., Fujita, I., Tanaka, K. (1995) Size and position invariance of neuronal responses in monkey inferotemporal cortex. J Neurophysiol. 1995 Jan;73(1):218-26. 
Jedlicka, P. (2017) Revisiting the Quantum Brain Hypothesis: Toward Quantum (Neuro)biology?. Front Mol Neurosci. 2017; 10: 366.

Leighton, R. (1959) Bose-Einstein Condensation and Superfluidity. Principles of Modern Physics, Mcgraw-Hill

Li, S. (2017) Rotation Invariance Neural Network. arXiv:1706.05534v1 [cs.CV]

Li, S., Long, G., Bai, F., Feng, S., Zheng, H. (2001). Quantum computing. PNAS October 9, 200198 (21) 11847-11848; https://doi.org/10.1073/pnas.191373698

Lloyd, S. (1996) Universal Quantum Simulators. Science 23 Aug 1996: Vol. 273, Issue 5278, pp. 10731078 DOI: $10.1126 /$ science.273.5278.1073

Lundholm, I., Rodilla, H., Wahlgren, W., Duelli, A., Bourenkov, G., Vukusic, J., Friedman, R., Stake, J., Schneider, T., Katona, G. (2015) THz radiation induces Frohlich condensate in protein. Structural Dynamics 2, 054702 (2015); https://doi.org/10.1063/1.4931825

Kwisthout, J. (2014). Minimizing Relative Entropy in Hierarchical Predictive Coding. In: van der Gaag L.C., Feelders A.J. (eds) Probabilistic Graphical Models. PGM 2014. Lecture Notes in Computer Science, vol 8754. Springer, Cham

Maldacena, J. (1997). The Large N Limit of Superconformal Field Theories and Supergravity.

Adv.Theor.Math.Phys.2:231-252,1998. 10.1023/A:1026654312961

Marshall, I. (1989). Consciousness and Bose-Einstein Condensates New Ideas in Psychology vol.7, pg.73-83

Martindale, D. (2005). One Face, One Neuron. Scientific American

Minkel, J. (2009) Strange but True: Superfluid Helium Can Climb Walls. Scientific American

Miyaji, M., Takayanagi, T. (2015) Surface/State Correspondence as a Generalized Holography.

arXiv:1503.03542v1 [hep-th]

Momeni, D., Mansoori, S., Myrzakulov, R. (2016) Holographic complexity in gauge/string

superconductors. Phys. Lett. B, 756 (2016), 354-357 .10.1016/j.physletb.2016.03.031

Moskowitz, C. (2016) Tangled Up in Spacetime. Scientific American

Musser, G. (2018) What Is Spacetime?. Nature

Nam, Y., Ross, N., Su, Y., Childs, A., Maslov, D. (2018) Automated optimization of large quantum circuits with continuous parameters | npj Quantum Information. npj Quantum Information

volume 4, Article number: 23 (2018)

NASA (2014) NASA - Universe 101. ONLINE

O'Donnell, R. (2015) QMA is thought to equal NP. ONLINE. Lecture 24: QMA: Quantum Merlin-Arthur

Perera, T., et. al. (2019) PC12 Pheochromocytoma Cell Response to Super High Frequency Terahertz Radiation from Synchrotron Source. Cancers 11(2):162 - January 2019. DOI: 10.3390/cancers11020162 
Renka, R. (2018) Quantum Circuits. ONLINE

Robertson, L. C. (2005). Neurobiology of attention. Neurobiology of Attention

2005, Pages 135-139. https://doi.org/10.1016/B978-012375731-9/50028-8

Rokicki, T., Kociemba, H., Davidson, M., Dethridge, J. (2014): The diameter of the Rubik's cube group is twenty. SIAM Rev. 56(4), 645-670

Rosenblatt, F. (1958) The perceptron: a probabilistic model for information storage and organization in the brain, Psychological Review 65 (6) 386.

Rufini, G. (2017) Consciousness and Algorithmic Information Theory. Neuroscience of Consciousness, Volume 2017, Issue 1, 1 January 2017, nix019, https://doi.org/10.1093/nc/nix019

Schmitt, A. (2014) Introduction to superfluidity. Lect. Notes Phys. 888, 1-155 (2014), ISBN 978-3-31907946-2

Schuld, M., Sinayskiy, I., Petruccione, F. (2014) Simulating a perceptron on a quantum computer. Physics Letters A, 379, pp. 660-663 (2015). 10.1016/j.physleta.2014.11.061

Shor, P. (1995) Polynomial-Time Algorithms for Prime Factorization and Discrete Logarithms on a Quantum Computer. SIAM J.Sci.Statist.Comput. 26 (1997) 1484. 10.1137/S0097539795293172

Spratling M. W. (2016). A Hierarchical Predictive Coding Model of Object Recognition in Natural Images. Cognitive computation, 9(2), 151-167. doi: 10.1007/s12559-016-9445-1

Sountsov, P., Santucci, D., Lisman, J. (2011) A biologically plausible transform for visual recognition that is invariant to translation, scale, and rotation. Front. Comput. Neurosci., 22 November 2011 | https://doi.org/10.3389/fncom.2011.00053

Sousa, R., Hermann, K., Conway, B. (2015) Striking individual differences in color perception uncovered by The Dress photograph. Curr Biol. 2015 Jun 29; 25(13): R545-R546. doi: 10.1016/j.cub.2015.04.053

Spartling, M. (2017). A Review of Predictive Coding Algorithms. Brain and Cognition, doi: 10.1016/j.bandc.2015.11.00.

Stiefel, K.M., Holcomb, A.O. (2014). The Claustrum - Structural, Functional, and Clinical Neuroscience:Chapter 6 - Neurocomputation and Coding in the Claustrum: Comparisons with the Pulvinar. The Claustrum.Structural, Functional, and Clinical Neuroscience. 2014, Pages 193-207

Stone, J. (2012) Vision and the Brain. The MIT Press

Susskind, L. (2014) Entanglement is not Enough. arXiv:1411.0690v1 [hep-th]

Susskind, L. (2014 B). ER=EPR, GHZ, and the Consistency of Quantum Measurements. arXiv:1412.8483v1 [hep-th]

Susskind, L. (2017) Dear Qubitzers, GR=QM. arXiv:1708.03040v1 [hep-th]

Susskind, L. (2018) Three Lectures on Complexity and Black Holes. arXiv:1810.11563v1 [hep-th] 
Tegmark, M. (1999) The importance of quantum decoherence in brain processes.

Phys.Rev.E61:4194-4206,2000. DOI: 10.1103/PhysRevE.61.4194

Van Raamsdonk, M. (2010). Building up spacetime with quantum entanglement. Gen.Rel.Grav.42:23232329,2010; Int.J.Mod.Phys.D19:2429-2435,2010. DOI:10.1007/s10714-010-1034-0

$10.1142 / \mathrm{S} 0218271810018529$

Vaziri, A., Plenio, M. (2010) Quantum coherence in ion channels: Resonances, Transport and

Verification. New J. Phys. 12, 085001 (2010). DOI: 10.1088/1367-2630/12/8/085001

von Ahn, L., Blum, M., Hopper, N., Langford, J. (2000) CAPTCHA: Telling Humans and Computers Apart Automatically. ONLINE

von der Malsburg, C. (1981). The correlation theory of brain function. [Intern. Rep., 81-2, MPI

Biophysikalische Chemie, Gottingen, Germany]

Wenger, T. (2012) Effective Field Theoretic Approach to Layered Superconductors in Holographic Superconductivity Effective Field Theoretic Approach to Layered Superconductors.

Wolchover, N. (2013) Signs of a Stranger, Deeper Side to Nature's Building Blocks. Quanta Magazine

Wolchover, N. (2014) New research suggests physicists, computers and brains employ the same procedure to tease out important features from among other irrelevant bits of data. Quanta Magazine

Wolchover, N. (2019) The same codes needed to thwart errors in quantum computers may also give the fabric of space-time its intrinsic robustness. Quanta Magazine

Xu, Y., Xiao, T., Zhang, J., Yang, K., Zhang, Z. (2014) Scale-Invariant Convolutional Neural Networks. arXiv:1411.6369v1 [cs.CV]

You, Y., Yang, Z., Qi, X. (2017) Machine Learning Spatial Geometry from Entanglement Features. Phys. Rev. B 97, 045153 (2018). DOI:10.1103/PhysRevB.97.045153

Zaanen, J., Sun, Y., Liu, Y., Schalm, K. (2015). Holographic Duality in Condensed Matter Physics. Cambridge University Press

Zhao, L., Hao, Y., Peng, R. (2014) Advances in the biological effects of terahertz wave radiation. Mil Med Res. 2014; 1: 26. doi: 10.1186/s40779-014-0026-x

Zhang, S. (2018) Complexity and phase transitions in a holographic QCD model. Nuclear Physics B Volume 929, April 2018, Pages 243-253. https://doi.org/10.1016/j.nuclphysb.2018.02.010 